\title{
Overview of the University of Pennsylvania's TIPSTER Project University of Pennsylvania
}

\author{
Breck Baldwin \\ Institute for Research \\ Thomas S. Morton \\ in Cognitive Science \\ Department of Computer \\ and Information Science \\ University of Pennsylvania University of Pennsylvania \\ \{breck, tsmorton, bagga\}@unagi.cis . upenn .edu
Amit Bagga
Department of Computer
Science
Duke University

\section{Introduction}

CAMP software has been used in a variety of areas, but at the end of TIPSTER it finishes as it startedas a coreference annotation system. The coreference output has been used to participate in MUC6 and MUC-7, served as the foundation for three types of summarization engines and been input to a cross-document coreference system for names and events. This document focuses on the most successful of these application, a query sensitive summarization system and a cross-document coreference system.

\section{Dynamic Coreference-Based Summarization}

We have developed a query-sensitive text summarization technology well suited for the task of determining whether a document is relevant to a query. Enough of the document is displayed for the user to determine whether the document should be read in its entirety. Evaluations indicate that summaries are classified for relevance nearly as well as full documents. This approach is based on the concept that a good summary will represent each of the topics in the query and is realized by selecting sentences from the document until all the phrases in the query which are represented in the summary are 'covered.' A phrase in the document is considered to cover a phrase in the query if it is coreferent with it. This approach maximizes the space of entities retained in the summary with minimal redundancy. The software is built upon the CAMP NLP system [3].

\section{Problem Statement}

Given the relative immaturity of summarization technologies and their evaluation, it is worthwhile to describe our approach in detail and the problems it is intended to solve. An important aspect of our technique is that we produce sentence extraction summaries which are constructed by selecting sentences from the source document. In addition, our summaries are focused on providing relevant information about a query. We feel that the current state-of-the-art techniques are better equipped to produce high quality query-sensitive summaries than generic summaries. Our goal is to produce 'indicative' summaries [5] which allow a user to determine whether the document is relevant to his or her query. The summary is not intended to replace the document or provide answers to questions directly but may have this effect.

Casting our technology in terms of a product, we see the application as an intermediate step between viewing entire documents and the output of an information retrieval engine. Instead of looking at either headlines or an entire document, the user would look at the summaries of the documents and then decide whether the document merited further reading.

\section{Approach}

We conducted a simple experiment with summaries produced in the TIPSTER summarization dry run [8]. For 5 queries with 200 documents each, we took the set of summaries produced by the 6 dryrun participants and retained only those summaries that were true-positives, i.e., the summary was judged 'relevant' and the full document was judged 'relevant'. Over all the queries, at least one of the six systems produced a true-positive summary for $96.6 \%$ of the documents, although no individual system performed nearly at that level. This meant that some existing technology produced a correct summary for almost every relevant document. Hence we viewed the problem as one of balancing the capabilities of our system to behave like 
the amalgamated system implicit in joined output. Based on this result we are confident that this class of summarization is tractable with current technologies and this has strongly motivated our design decisions.

Upon encountering a query like "Reporting on possibility of and search for extra-terrestrial life/intelligence.", we assume that the user has defined a class of actions, ideas, and/or entities that he or she is interested in. The job of an information retrieval engine is to find instantiations of those classes in text documents in some database. We view summarization as an additional step in this process where we attempt to present the user with the smallest collection of sentences in the document that instantiate the user specified classes and do not mislead the user about the overall content of the document. By doing so, we can greatly shorten the amount of the document that the user must read in order to determine whether the document is relevant for the user's needs.

Just as information retrieval algorithms approximate document relatedness by examining various string matchings between the query and the text, we approximate certain classes of coreference between the query and the text by examining linguistic information. These coreference relations include identity of reference and part-whole relations for nominal and verbal phrases. ${ }^{1}$ This moves us a step closer to reasoning at a more appropriate level of generalization, for summarization, which is still technologically feasible. Below are examples indicating the classes of relatedness that we are trying to capture.

\section{The identity relation between the query and the document}

Noun phrase coreference is the best understood class of relations that we compute. For example, there is coreference between 'Federal Emergency Management Agency' in the query and the acronym 'FEMA' in the document below:

Query: What is the main function of the Federal Emergency Management Agency and the funding level provided to meet emergencies?

Document: ...FEMA agrees that "finetuning" is needed to the 1974 act establishing a coordinated federal program to prepare for

\footnotetext{
${ }^{1}$ It is not clear whether more sophisticated annotations are appropriate for information retrieval, and perhaps more to the point, it is not clear that there are sufficient resources to process 2 GB collections of data.
}

and respond to hurricanes, tornadoes, storms and floods. ...

Since these noun phrases refer to the same entity in the world, sentences that mention the organization would be particularly valuable in a summary. This class of coreference can include people, companies and objects such as automobiles or aluminum siding. It need not be restricted to proper nouns as it is possible to refer to an entity using common nouns, i.e. 'the agency' and pronouns.

Identity also holds between events mentioned in the query and document. Sometimes the event that a query describes is the best indicator of what document should be retrieved, and correspondingly what sentences are appropriate for a summary. Consider the following:

Query: A relevant document will provide new theories about the 1960's assassination of President Kennedy.

Document: ...The House Assassinations Committee concluded in 1978 that Kennedy was "probably" assassinated as the result of a conspiracy involving a second gunman, a finding that broke from the Warren Commission's belief that Lee Harvey Oswald acted alone in Dallas on Nov. 22, 1963. ...

The noun phrase 'the 1960's assassination' refers to an event, which is the same as the one referred to in the document with the verb 'assassinated'. Note also that there is coreference between 'President Kennedy' and 'Kennedy' in the document.

\section{The part-whole relation between the query and the document}

In addition to the identity relation, phrases in a text which refer to parts of an entity or concept mentioned in the query will likely provide useful information, and therefore should be included in a summary. Finding these relations in in general is beyond the scope of this paper, however, our approximation of a subclass of these relations proved helpful for a number of queries.

A strong example of the part-whole relation occurs when a country is mentioned in the query and a province or city within that country is mentioned in the document. For example:

Query: Document will discuss efforts by the black majority in South Africa to overthrow domination by the white minority government. 
Document: About 90 soldiers have been arrested and face possible death sentences stemming from a coup attempt in Bophuthatswana, ... Rebel soldiers staged the takeover bid Wednesday, detaining homeland President Lucas Mangope. ...

Bophuthatswana is inside South Africa, and sentences that mention it are clearly good candidates for inclusion in a summary.

We also consider part-whole relations between events as in the relation between 'overthrow' and 'staged' and 'detained'. Those events are sub-parts of overthrow events, and as such, sentences that contain sub-parts of the events are reasonable candidates for inclusion in summaries.

\section{Implementation}

The summarization technique was developed within the CAMP NLP framework. This system provides an integrated environment in which to access many levels of linguistic information as well as world knowledge. Its main components include: named entity recognition, tokenization, sentence detection, part-of-speech tagging, morphological analysis, parsing, argument detection, and coreference resolution. Many of the techniques used for these tasks perform at or near the state of the art and are described in more depth in $[16,12,11,9,6,2,3]$. The system produces coreference annotated documents which serve as the input to the summarization algorithm.

\section{Relating the query to the document}

The relationships discussed previously are approximated via a series of associations between tokens in the query, headline, and the body of the document. Event references are captured by associating verbs or nominalizations in the query with verbs and nominalizations in the document.

Given three verbal forms $v_{1}$ in the query, $v_{2}$ in the document, and $v_{3}$ in the set of all verbal forms, where a verbal form is the morphological root of a verb or the verb root corresponding to a nominalization, $v_{1}$ is associated with $v_{2}$ if at least one of the following criteria are met:

1. $\left(v_{1} \neq v_{2}\right) \wedge p\left(v_{1}, v_{2}\right) /\left(p\left(v_{1}\right) p\left(v_{2}\right)\right) \geq 5$

2. $\left(v_{1}=v_{2}\right) \wedge\left(\exists v_{3} \neq v_{1} \mid p\left(v_{1}, v_{3}\right) / p\left(v_{1}\right) p\left(v_{3}\right) \geq 5\right)$

3. $\left(v_{1}=v_{2}\right) \wedge\left(\left(\operatorname{subject}\left(v_{1}\right)=\operatorname{subject}\left(v_{2}\right)\right) \vee\right.$ $\left.\left(\operatorname{object}\left(v_{1}\right)=\operatorname{object}\left(v_{2}\right)\right)\right)$
Here $p\left(v_{i}\right)$ is the probability that $v_{i}$ occurs in a document and $p\left(v_{i}, v_{j}\right)$ is the probability that $v_{i}$ and $v_{j}$ occur in the same document. These probabilities are based on frequencies gathered from approximately 45,000 Wall Street Journal articles. Criterion 1 is a measure of mutual information between two verbs. Criterion 2 is used to rule out frequently occurring verbs such as "be" and "make". Criterion 3 allows for verbs which are ruled out by criterion 2 to be associated when additional context is available. This is important since some queries only contain verbal forms which are ruled out by criterion 2.

Relationships between proper nouns are made on the basis of string matches, acronym matching, and dictionary lookup. Acronyms are determined either through a table lookup or an appositive construction occurring in the document which designates the acronym for a specific proper noun. A proper noun in the query is considered associated with a proper noun in the document if it matches the string or acronym of the proper noun in the document or it appears in the definition of the proper noun in the document. A reverse dictionary lookup often allows cities to be associated with the country they are in.

A token in the query which is a lowercase noun or adjective is associated with any token in the document which matches its morphological root and part of speech.

Tokens which occur in the headline are associated with tokens in the document body using the same criteria as the query, with the exclusion of the dictionary lookup. The dictionary lookup was excluded because the headline will likely use the same lexicalization of a proper noun as that used in a document. This is less likely to be the case with the query.

\section{Selecting a sentence}

The associations discussed in the previous section are used to rank and select sentences from the document. Every token in the document which is associated with the same token in the query or headline is considered to be in the same coreference chain. A sentence which contains any token in a given coreference chain is said to cover that chain.

The following scores are computed for each sentence in the document:

1. The number of coreference chains from the query which are covered by the sentence and haven't been covered by a previously selected sentence. 
2. The number of noun coreference chains from the query which are covered by the sentence and the number of verbal terms in the sentence which are chained to the query.

3. The number of coreference chains from the headline which are covered by the sentence and haven't been covered by a previously selected sentence.

4. The number of noun coreference chains from the headline which are covered by the sentence and the number of verbal terms in the sentence which are chained to the headline.

5. The number of coreference chains which are covered by the sentence and haven't been covered by a previously selected sentence.

6. The number of noun coreference chains which are covered by the sentence.

7. The index of the sentence in the document; sentences are sequentially numbered.

The sentences are sorted based on the above scores, where the ith scoring criteria is only considered in case of a tie for all criteria less than $i$. Scores 1-6 are ranked in descending order while score 7 is ranked in ascending order. The top-ranked sentence is selected, and scores 1,3 , and 5 are recomputed in order to select the next sentence. Selection halts when all coreference chains in the query have been covered and the summary contains at least 4 sentences.

Scores 1 and 2 are used to select sentences which are related to the query. Scores 3 and 4 are motivated by documents which have 1 or 2 sentences which appear related to the query but if presented alone would give a false impression of the true content of the document. Thus sentences related to the headline are presented to provide additional background. Consider the following example:

Query: What evidence is there of paramilitary activity in the U.S.?

Summary: ... Last month the extremists used rocket-propelled grenades for the first time in three attacks on police and paramilitary units.$$
\text { ... }
$$

This sentence was selected because it contains tokens which are in coreference chains with tokens in the query; however, alone it is potentially misleading because the place of the attack is not mentioned. This ambiguity is resolved when the following sentence is selected because it is well associated with the headline.
Summary: ...Sikh militants may have acquired one or two U.S.-made Stinger antiaircraft missiles and hidden them inside the Golden Temple, the Sikh faith's holiest shrine, Punjab police officials said Saturday....

This provides enough background information for the reader to realize that the para-military activity is not taking place in the U.S. and thus that the document is irrelevant to the query.

Likewise, scores 5 and 6 act similarly to 3 and 4 for documents which do not contain a headline. We found this particularly important for advertisements which often don't state a product or company name in the beginning of the document, but will repeat these names numerous times throughout the document.

\section{Generating the summary}

Once sentences have been selected, they are presented in the order they occurred in the document. Pronouns which do not have a referent in the previous sentence of the summary are filled with a more descriptive string whenever a referent can be determined. If space is of concern, prepositional phrases attached to nouns (which are not nominalizations), appositives, conjoined noun phrases and relative clauses are removed, provided they contain no tokens associated with the query or the headline. Since determining pronoun referents and the selection of clauses for removal are subject to errors, filled pronouns are placed in square brackets and removed clauses are replaced with an ellipsis to indicate to the reader that the original text has been modified.

\section{Example summary}

An example summary which demonstrates many of the features of our system appears below. It has been constrained to be approximately $10 \%$ of the original document length, so it is not representative of the summaries used in the evaluation, but it contains examples of the of both pronoun filling and clause deletion.

The last sentence in the summary was selected first because the tokens "death", "sentence", "kill", and "term" were associated with the nominalization "punishment". The stranded pronoun "it" has also been filled. Sentence 2 was selected next because of the match-up between the verb "is" and the object "deterrent" in the document and the query. Finally, the first sentence was chosen because there is another mention of the prison name "Marion" in the document. This summary differs 
from the one generated when the $10 \%$ length constraint is not imposed, because some higher ranked sentences were passed over since their inclusion would have exceeded the length restriction.

Query: Is there data available to suggest that capital punishment is a deterrent to crime?

Summary: "Marion is basically the end of the line," Bogdan said.

... There is no deterrent ... to keep them from doing this again.

Additionally, [the pending Senate bill] would create five new death penalty offenses: murder by a federal inmate serving a life sentence; drug kingpins in a continuing criminal enterprise even if no murders occur; drug kingpins who try to kill to obstruct justice; drug felons who unintentionally kill with aggravated recklessness; and people who kill with a firearm during a violent ... crime.

\section{Evaluation}

In order to evaluate our summarization algorithm, we selected 10 unseen queries from the Text REtrieval Conference (TREC) document collection. Summaries were generated for 200 documents, 20 per query, and assessors ${ }^{2}$ were asked to make relevance judgments based on the summaries. A document was considered relevant if it contained the information requested in the query or if the assessor believed that the full document would likely contain this information. The relevance judgments were then compared to those made by the TREC assessors using the full document. This comparison places a summary in one of the following categories:

- $\mathrm{a}=$ judged relevant, full document is relevant

- $b=$ judged relevant, full document is irrelevant

- $c=$ judged irrelevant, full document is relevant

- $d=$ judged irrelevant, full document is irrelevant

Precision, recall, and accuracy are then computed as follows:

$$
\begin{gathered}
\text { precision }=a /(a+b) \\
\text { recall }=a /(a+c) \\
\text { accuracy }=(a+d) /(a+b+c+d)
\end{gathered}
$$

Compression is computed over the number of non-whitespace characters in the summary and the original document. Here compression is defined as

\footnotetext{
${ }^{2}$ Each author served as an assessor making judgments for 100 documents across 10 queries.
}

the percentage of the document that was not included in the summary:

$$
\text { compression }=\frac{\left(\text { length }_{\text {document }}-\text { length }_{\text {summary }}\right)}{\text { length document }}
$$

The results from our experiment are shown in the following table:

\begin{tabular}{|l|c|c|}
\hline Precision & $82.8 \%$ & $101 /(101+21)$ \\
\hline Recall & $77.7 \%$ & $101 /(101+29)$ \\
\hline Compression & $82.8 \%$ & $(704686-121272) / 704686$ \\
\hline Accuracy & $75.0 \%$ & $(101+49) / 200$ \\
\hline
\end{tabular}

A second evaluation on 910 documents was performed for [5]. These results superficially appear significantly worse than those from the initial evaluation however a more careful analysis (provided in the discussion section) shows that they are in fact similar to the results of the previous evaluation.

\begin{tabular}{|l|c|c|}
\hline Precision & $80.3 \%$ & $322 /(322+79)$ \\
\hline Recall & $57.6 \%$ & $322 /(322+237)$ \\
\hline Compression & $83.0 \%$ & \\
\hline Accuracy & $65.3 \%$ & $(322+272) / 910$ \\
\hline
\end{tabular}

\section{Discussion}

We view the results of the first evaluation as promising in that they compare favorably with inter-assessor consistency using the entire document. [15] reports unanimous relevance judgments by three assessors for $71.7 \%$ of the documents. Interpolating this figure to two assessors yields an $80.1 \%$ agreement figure. Using summaries which on average are only $17.2 \%$ of the original document, our assessors matched the TREC assessors for $75.0 \%$ of the documents.

The second evaluation yielded a much lower recall figure while precision remained comparable. This, however, is also the case when the same assessors judgments on the full documents are compared to those of the TREC assessors. These results are as follows:

\begin{tabular}{|l|r|c|}
\hline Precision & $83.5 \%$ & $167 /(167+33)$ \\
\hline Recall & $63.5 \%$ & $167 /(167+96)$ \\
\hline Compression & $100.0 \%$ & \\
\hline Accuracy & $69.3 \%$ & $(167+124) / 420$ \\
\hline
\end{tabular}

We view these results as favorable as well since our accuracy is $65.3 \%$ using $17.0 \%$ of the document on average compared to $69.3 \%$ accuracy using the entire document. The discrepancy between the two evaluations appears to be based on the assessors in the second evaluation using a stricter criteria for relevance than that used by the previous evaluation's assessors or the TREC assessors.

It was noted after the first evaluation that different criteria for relevance accounted for some of 
the disagreement between our assessors and the TREC assessors. Many documents considered relevant were marked as irrelevant due to different notions of relevance and not because the summary failed to provide material on which to base a correct decision. These difficulties only hinder the evaluation of a summary system and not its use in an application, since a user will have a clear idea of his or her intentions when determining a document's relevance.

As we mentioned previously, our approach has been to balance methods of relating the query to sentences in the document. The nearly $100 \%$ recall of the dry-run summaries encouraged us, and we even used the output of those summaries to provide a test-bed for evaluating our summaries. Although we never actively sought to emulate aspects of other systems directly, our final algorithm does share some basic ideas and approaches from those systems. Some of the similarities are listed below:

In [4], they eliminate redundant information from summaries by classifying sentences according to Maximal Marginal Relevance (MMR). MMR ranks text chunks according to their dissimilarity to one another. Summaries can then be produced with sentences that are maximally dissimilar, thereby increasing the likelihood that distinguishing information will be in the summary. One can view our coverage requirement for terms in the query as an attempt to pick dissimilar sentences from the document. Instead of MMR, we use the fact that a sentence which does not contain redundantly referring phrases to the query is more highly ranked than a sentence that does.

Our individual sentence scoring algorithm shares some properties with [14]. Their approach includes scores for anaphoric density, string equivalence with the title or headline of a document, and position of the sentence in the document. However, we do not take advantage of overt cues for summary sentences, such as 'in summary' or 'in conclusion', nor do we use temporal information in generating a summary.

Like many systems, we do a form of word expansion in attempting to relate the query to the document. However, the fact that we restrict expansion to proper nouns and verbs and their nominalizations is notable. We found this limited set of expansions restricts the relations between the text and the query well and also fits within the framework of part-whole relations in coreference. We did not consider part-whole relations for common nouns, because in practice we have not had very good results limiting over-generation in that domain.

In the next section we discuss a novel technology for cross document coreference. Like the summarization system just discussed, it takes within document coreference annotated text, produces summaries in a very similar form to the above, and individuates entities based on the similarity of the summaries produced.

\section{Cross-document Coreference}

Cross-document coreference occurs when the same person, place, event, or concept is discussed in more than one text source. Computer recognition of this phenomenon is important because it helps break "the document boundary" by allowing a user to examine information about a particular entity from multiple text sources at the same time. In particular, resolving cross-document coreferences allows a user to identify trends and dependencies across documents. Cross-document coreference can also be used as the central tool for producing summaries from multiple documents, and for information fusion, both of which have been identified as advanced areas of research by the TIPSTER Phase III program. Cross-document coreference was also identified as one of the potential tasks for the Sixth Message Understanding Conference (MUC-6) but was not included as a formal task because it was considered too ambitious [10].

In this paper we describe a highly successful cross-document coreference resolution algorithm which uses the Vector Space Model to resolve ambiguities between people having the same name. In addition, we also describe a scoring algorithm for evaluating the cross-document coreference chains produced by our system and we compare our algorithm to the scoring algorithm used in the MUC-6 (within document) coreference task.

\section{Cross-Document Coreference: The Problem}

Cross-document coreference is a distinct technology from Named Entity recognizers like IsoQuest's NetOwl and IBM's Textract because it attempts to determine whether name matches are actually the same individual (not all John Smiths are the same). Neither NetOwl or Textract have mechanisms which try to keep same-named individuals distinct if they are different people.

Cross-document coreference also differs in substantial ways from within-document coreference. Within a document there is a certain amount of 
consistency which cannot be expected across documents. In addition, the problems encountered during within document coreference are compounded when looking for coreferences across documents because the underlying principles of linguistics and discourse context no longer apply across documents. Because the underlying assumptions in cross-document coreference are so distinct, they require novel approaches.

\section{Architecture and the Methodology}

Figure 1 shows the architecture of the crossdocument system developed. The system is built upon the University of Pennsylvania's within document coreference system, CAMP, which participated in the Seventh Message Understanding Conference (MUC-7) within document coreference task.

Our system takes as input the coreference processed documents output by CAMP. It then passes these documents through the SentenceExtractor module which extracts, for each document, all the sentences relevant to a particular entity of interest. The VSM-Disambiguate module then uses a vector space model algorithm to compute similarities between the sentences extracted for each pair of documents.

Details about each of the main steps of the crossdocument coreference algorithm are given below.

- First, for each article, CAMP is run on the article. It produces coreference chains for all the entities mentioned in the article. For example, consider the two extracts in Figures 2 and 4. The coreference chains output by CAMP for the two extracts are shown in Figures 3 and 5.

- Next, for the coreference chain of interest within each article (for example, the coreference chain that contains "John Perry"), the Sentence Extractor module extracts all the sentences that contain the noun phrases which form the coreference chain. In other words, the SentenceExtractor module produces a "summary" of the article with respect to the entity of interest. These summaries are a special case of the query sensitive techniques being developed at Penn using CAMP. Therefore, for doc.36 (Figure 2), since at least one of the three noun phrases ("John Perry," "he," and "Perry") in the coreference chain of interest appears in each of the three sentences in the extract, the summary produced by SentenceExtractor is the extract itself. On the other hand, the summary produced by SentenceExtractor for the coreference chain of interest in
John Perry, of Weston Golf Club, announced his resignation yesterday. He was the President of the Massachusetts Golf Association. During his two years in office, Perry guided the $M G A$ into a closer relationship with the Women's Golf Association of Massachusetts.

Figure 2: Extract from doc.36

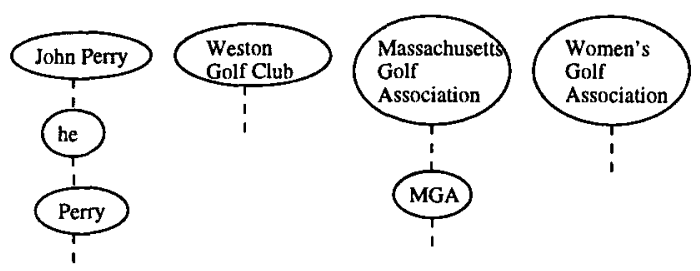

Figure 3: Coreference Chains for doc.36

doc. 38 is only the first sentence of the extract because the only element of the coreference chain appears in this sentence.

- For each article, the VSM-Disambiguate module uses the summary extracted by the SentenceExtractor and computes its similarity with the summaries extracted from each of the other articles. Summaries having similarity above a certain threshold are considered to be regarding the same entity.

\section{University of Pennsylvania's CAMP System}

The University of Pennsylvania's CAMP system resolves within document coreferences for several different classes including pronouns, and proper names [7]. It ranked among the top systems in the coreference task during the MUC-6 and the MUC-7 evaluations.

The coreference chains output by CAMP enable us to gather all the information about the entity of interest in an article. This information about the entity is gathered by the SentenceExtractor module and is used by the VSM-Disambiguate module for disambiguation purposes. Consider the extract for doc.36 shown in Figure 2. We are able to include the fact that the John Perry mentioned in this article was the president of the Massachusetts Golf Association only because CAMP recognized that the "he" in the second sentence is coreferent with "John Perry" in the first. And it is this fact which actually helps VSM-Disambiguate decide that the 


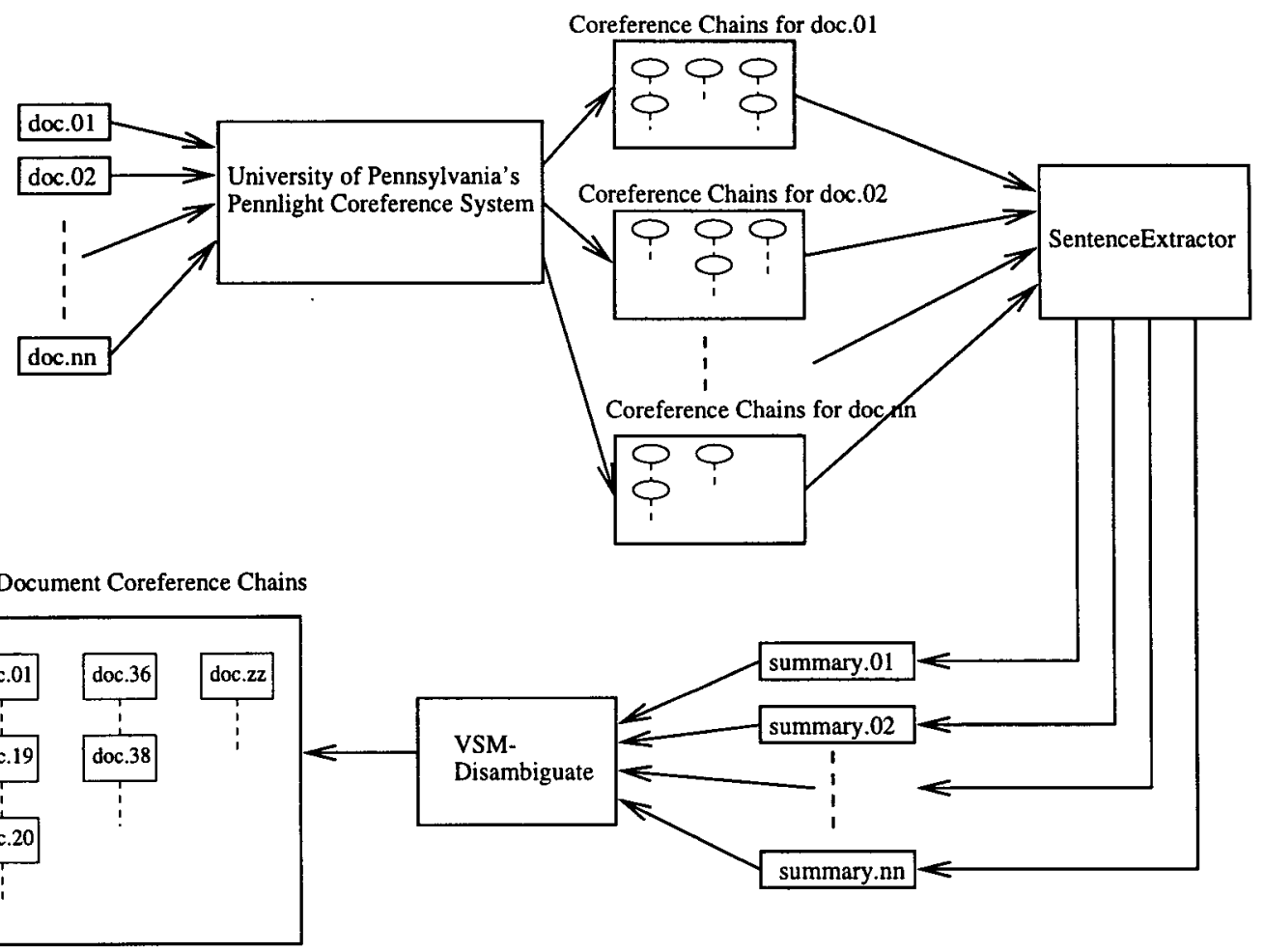

Figure 1: Architecture of the Cross-Document Coreference System

Oliver "Biff" Kelly of Weymouth succeeds John Perry as president of the Massachusetts Golf Association. "We will have continued growth in the future," said Kelly, who will serve for two years. "There's been a lot of changes and there will be continued changes as we head into the year 2000."

Figure 4: Extract from doc.38
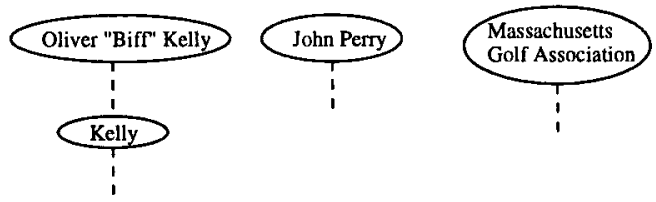

Figure 5: Coreference Chains for doc.38

two John Perrys in doc. 36 and doc. 38 are the same person.

\section{The Vector Space Model}

The vector space model used for disambiguating entities across documents is the standard vector space model used widely in information retrieval [13]. In this model, each summary extracted by the SentenceExtractor module is stored as a vector of terms. The terms in the vector are in their morphological root form and are filtered for stop-words (words that have no information content like $a$, the, of, $a n, \ldots)$. If $S_{1}$ and $S_{2}$ are the vectors for the two summaries extracted from documents $D_{1}$ and $D_{2}$, then their similarity is computed as:

$$
\operatorname{Sim}\left(S_{1}, S_{2}\right)=\sum_{\text {common terms } t_{j}} w_{1 j} \times w_{2 j}
$$

where $t_{j}$ is a term present in both $S_{1}$ and $S_{2}, w_{1 j}$ is the weight of the term $t_{j}$ in $S_{1}$ and $w_{2 j}$ is the weight of $t_{j}$ in $S_{2}$.

The weight of a term $t_{j}$ in the vector $S_{i}$ for a summary is given by:

$$
w_{i j}=\frac{t f \times \log \frac{N}{d f}}{\sqrt{s_{i 1}^{2}+s_{i 2}^{2}+\ldots+s_{i n}^{2}}}
$$

where $t f$ is the frequency of the term $t_{j}$ in the summary, $N$ is the total number of documents in the collection being examined, and $d f$ is the number of documents in the collection that the term $t_{j}$ occurs in. $\sqrt{s_{i 1}^{2}+s_{i 2}^{2}+\ldots+s_{i n}^{2}}$ is the cosine normalization factor and is equal to the Euclidean length of the vector $S_{i}$. 
The VSM-Disambiguate module, for each summary $S_{i}$, computes the similarity of that summary with each of the other summaries. If the similarity computed is above a pre-defined threshold, then the entity of interest in the two summaries are considered to be coreferent.

\section{Experiments}

The cross-document coreference system was tested on a highly ambiguous test set which consisted of 197 articles from 1996 and 1997 editions of the New York Times. The sole criteria for including an article in the test set was the presence or the absence of a string in the article which matched the "/John.*?Smith/" regular expression. In other words, all of the articles either contained the name John Smith or contained some variation with a middle initial/name. The system did not use any New York Times data for training purposes. The answer keys regarding the cross-document chains were manually created, but the scoring was completely automated.

\section{Analysis of the Data}

There were 35 different John Smiths mentioned in the articles. Of these, 24 of them only had one article which mentioned them. The other 173 articles were regarding the 11 remaining John Smiths. The background of these John Smiths , and the number of articles pertaining to each, varied greatly. Descriptions of a few of the John Smiths are: Chairman and CEO of General Motors, assistant track coach at UCLA, the legendary explorer, and the main character in Disney's Pocahontas, former president of the Labor Party of Britain.

\section{Results}

Figure 6 shows the precision, recall, and F-Measure (with equal weights for both precision and recall) using the B-CUBED scoring algorithm. The Vector Space Model in this case constructed the space of terms only from the summaries extracted by SentenceExtractor. In comparison, Figure 7 shows the results (using the B-CUBED scoring algorithm) when the vector space model constructed the space of terms from the articles input to the system (it still used the summaries when computing the similarity). The importance of using CAMP to extract summaries is verified by comparing the highest $\mathrm{F}$ Measures achieved by the system for the two cases. The highest $\mathrm{F}$-Measure for the former case is $84.6 \%$ while the highest F-Measure for the latter case is $78.0 \%$. In comparison, for this task, named-entity

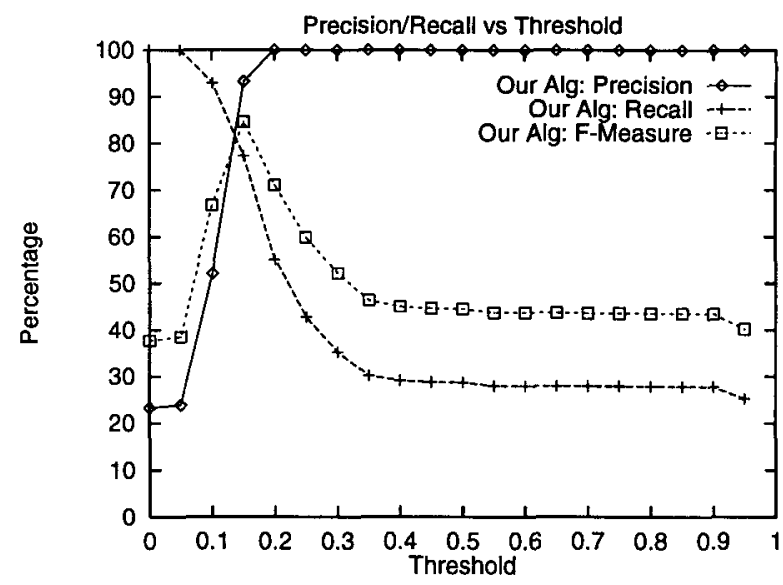

Figure 6: Precision, Recall, and F-Measure Using the B-CUBED Algorithm With Training On the Summaries

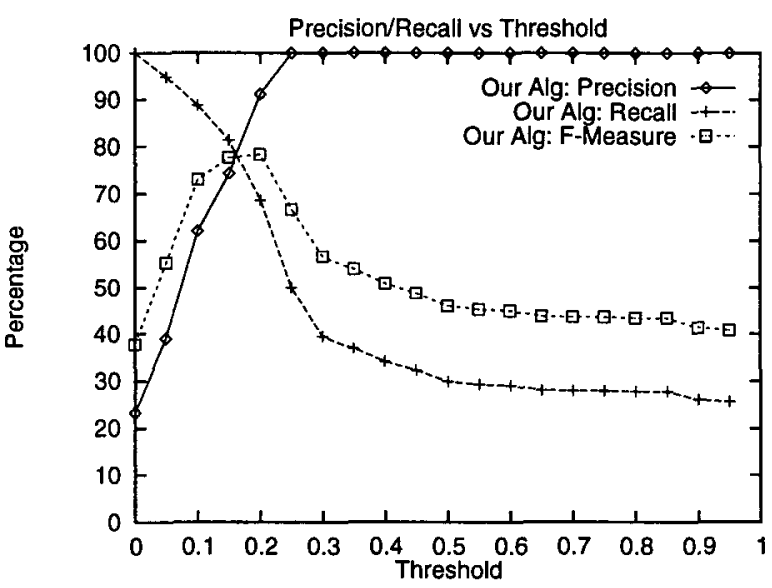

Figure 7: Precision, Recall, and F-Measure Using the B-CUBED Algorithm With Training On Entire Articles

tools like NetOwl and Textract would mark all the John Smiths the same. Their performance using our scoring algorithm is $23 \%$ precision, and $100 \%$ recall.

Figures 8 and 9 show the precision, recall, and F-Measure calculated using the MUC scoring algorithm. Also, the baseline case when all the John Smiths are considered to be the same person achieves $83 \%$ precision and $100 \%$ recall. The high initial precision is mainly due to the fact that the MUC algorithm assumes that all errors are equal.

We have also tested our system on other classes of cross-document coreference like names of companies, and events. Details about these experiments can be found in [1]. 


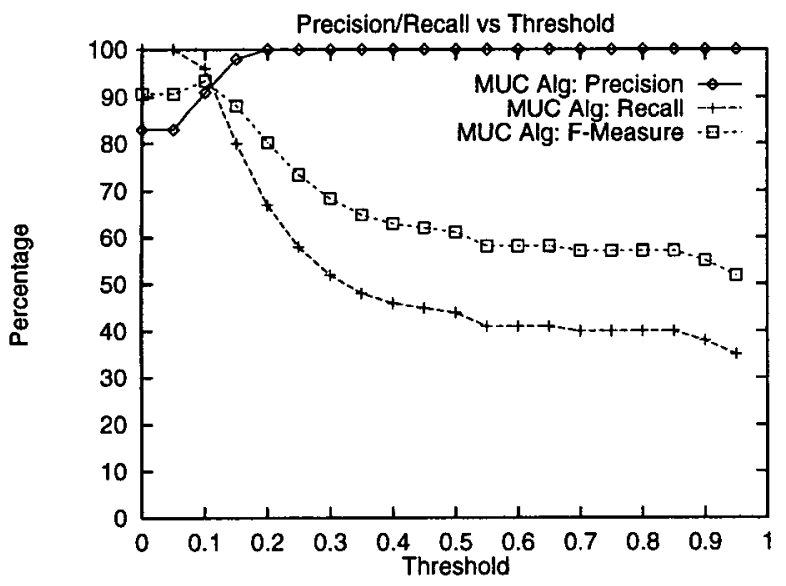

Figure 8: Precision, Recall, and F-Measure Using the MUC Algorithm With Training On the Summaries

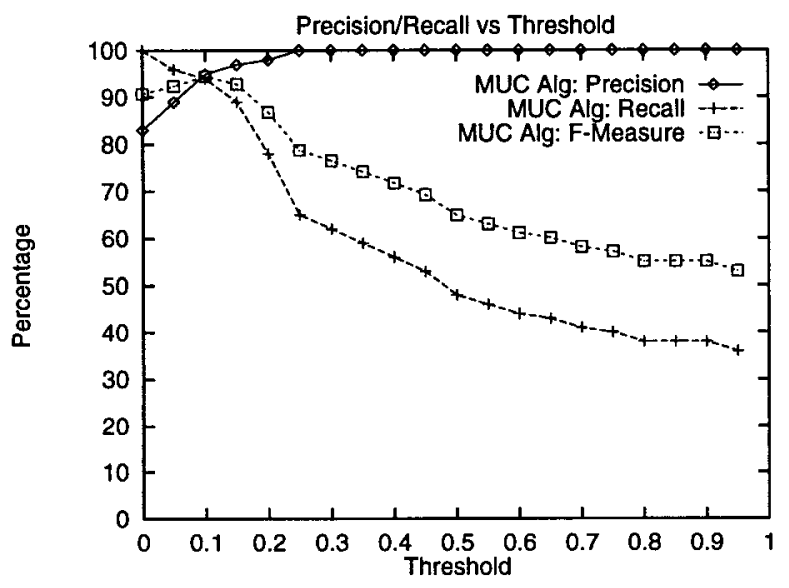

Figure 9: Precision, Recall, and F-Measure Using the MUC Algorithm With Training On Entire Articles

\section{Conclusions}

The TIPSTER phase III program has allowed us to explore some of the potential application areas of coreference annotation. We have reported on our strongest results, a summarization system and a cross-document coreference system for names.

The query-sensitive text summarization system is nearly as effective as full text documents for determining whether a document is relevant to the query. The system uses a limited class of coreference-based relations between the query and the document to select sentences which represent instantiations of entities, events, or concepts articulated in the query.

As a novel research problem, cross document coreference provides an different perspective from related phenomenon like named entity recognition and within document coreference. Our system takes summaries about an entity of interest and uses various information retrieval metrics to rank the similarity of the summaries. We found it quite challenging to arrive at a scoring metric that satisfied our intuitions about what was good system output v.s. bad, but we have developed a scoring algorithm that is an improvement for this class of data over other within document coreference scoring algorithms. Our results are quite encouraging with potential performance being as good as $84.6 \%$ (F-Measure).

\section{Future Goals}

Central to the future of this research program is the CAMP software system. We are continually refining and extending the software to better capture the coreference relations that we need and to reduce genre dependent aspects of the system. We are currently exploring visualization interfaces to both within and cross-document coreference which we believe will provide strong motivation for importance of corefence annotation of free text databases. In addition, we are interested in generating cross-document summaries based on similar techniques to our within document summarization system.

\section{References}

[1] Bagga Amit and Breck Baldwin. How much processing is required for cross-document coreference? In The First International Conference on Language Resources and Evaluation on Linguistics Coreference, Granada, Spain, 1998.

[2] Breck Baldwin. CogNIAC: High precision coreference with limited knowledge and linguistic resources. In Proceedings of the $A C L$ Workshop on Operational Factors in Practical, Robust Anaphora resolution for Unrestricted Texts, pages 38-45, Madrid, Spain, June 1997.

[3] Breck Baldwin, Christine Doran, Jeffrey C. Reynar, Michael Niv, B. Srinivas, and Mark Wasson. EAGLE: An extensible architecture for general linguistic engineering. In Proceedings of RIAO-97, Montreal, 1997.

[4] Michael Bett and Jade Goldstein. Automated query-relevant document summarization. In Proceedings of Tipster Text Phase III 12-Month Workshop, 1997. 
[5] Michael Chrzanowski, Therese Firmin, Lynette Hirschman, David House, Inderjeet Mani, Leo Obrst, Sara Shelton, Beth Sundheim, and Sandra Wagner. (SUMMAC) call for participation. http://www.tipster.org/summcall.htm, January 1998.

[6] Michael John Collins. A New Statistical Parser Based on Bigram Lexical Dependencies. In Proceedings of the 34th Annual Meeting of the $A C L, 1996$.

[7] Baldwin Breck et al. University of pennsylvania: Description of the university of pennsylvania system used for muc-6. In Proceedings of the Sixth Message Understanding Conference (MUC-6), pages 177-191, 1995.

[8] Therese Hand. Tipster summarization evaluation task:dry-run evaluation results. In Proceedings of Tipster Text Phase III 12-Month Workshop, 1997.

[9] Daniel Karp, Yves Schabes, Martin Zaidel, and Dania Egedi. A freely available wide coverage morphological analyzer for english. In Proceedings of the 15th International Conference on Computational Linguistics, 1994.

[10] Ralph Grishman. Whither Written Language Evaluation? In Proceedings of the Human Language Technology Workshop, 1994.

[11] Adwait Ratnaparkhi. A Maximum Entropy Part of Speech Tagger. In Eric Brill and Kenneth Church, editors, Conference on Empirical Methods in Natural Language Processing, University of Pennsylvania, May 17-18 1996.

[12] Jeffrey C. Reynar and Adwait Ratnaparkhi. A maximum entropy approach to identifying sentence boundaries. In Proceedings of the Fifth Conference on Applied Natural Language Processing, pages 16-19, Washington, D.C., April 1997.

[13] Gerard Salton. Automatic Text Processing: The Transformation, Analysis, and Retrieval of Information by Computer. Addison-Wesley, 1989.

[14] Tomek Strzalkowski, Fang Lin, Jin Wang, Langdon White, and Bowden Wise. Natural language information retrieval and summarization. In Proceedings of Tipster Text Phase III 12-Month Workshop, 1997.
[15] Ellen M. Voorhees and Donna Harman. Overview of the fifth Text REtrieval Conference (TREC-5). In Proceedings of the Fifth Text REtrieval Conference (TREC-5), pages 1-28. NIST 500-238, 1997.

[16] Nina Wacholder, Yael Ravin, and Misook Choi. Disambiguation of proper names in text. In Proceedings of the Fifth Conference on Applied Natural Language Processing, May 1997. 